\title{
Scanning tunneling microscopy and spectroscopy studies of graphite edges
}

\author{
Y. Niimi, T. Matsui, H. Kambara, K. Tagami, M. Tsukada, and Hiroshi Fukuyama* \\ Department of Physics, University of Tokyo, 7-3-1 Hongo Bunkyo-ku, Tokyo 113-0033, Japan
}

(Dated: April 5, 2004)

\begin{abstract}
We studied experimentally and theoretically the electronic local density of states (LDOS) near single step edges at the surface of exfoliated graphite. In scanning tunneling microscopy measurements, we observed the $(\sqrt{3} \times \sqrt{3}) R 30^{\circ}$ and honeycomb superstructures extending over $3-4 \mathrm{~nm}$ both from the zigzag and armchair edges. Calculations based on a density-functional derived nonorthogonal tight-binding model show that these superstructures can coexist if the two types of edges admix each other in real graphite step edges. Scanning tunneling spectroscopy measurements near the zigzag edge reveal a clear peak in the LDOS at an energy below the Fermi energy by $20 \mathrm{meV}$. No such a peak was observed near the armchair edge. We concluded that this peak corresponds to the "edge state" theoretically predicted for graphene ribbons, since a similar prominent LDOS peak due to the edge state is obtained by the first principles calculations.
\end{abstract}

PACS numbers: 61.16.Ch, 61.72.Ff, 73.20.At

\section{INTRODUCTION}

Graphite is one of the most extensively studied materials both experimentally and theoretically. Recently, electronic properties of graphite nanostructures, such as graphite edges, have been attracting much attention in view not only of basic research but also of applications. For example, porous carbons which consist an assembly of minute graphite fragments and naturally of a large amount of graphite edges have extremely high specific surface area [1, 2].

Topologically, there are two types of edges in singlelayer graphite sheet (graphene), i.e., zigzag and armchair edges (see Fig. 11). Fujita et al. [3] were the first to predict the existence of the specific electronic states localized only at the zigzag edge from the tight binding band calculations for graphene ribbons. On the other hand, it does not appear at the armchair edge. The flat band nature of this "edge state" results in a peak in the local density of states (LDOS) at the Fermi energy $\left(E_{F}\right)$. When the ribbon width is large enough, the contribution of the edge state to the total density of states should be negligibly small. However, this could be measurably large if we survey the LDOS, for example, with the scanning tunneling spectroscopy (STS) technique in the vicinity of zigzag edge. It is also predicted that a similar edge state appears at zigzag edges of multi-layer $\alpha \beta$ stacking ribbons [4]. This suggests that the edge state should be potentially observed at step edges on bulk graphite surfaces.

In the previous STS measurements [5, [6], a broad maximum near $E_{F}$ in the tunnel spectrum was reported to appear near circular edges of graphite nanopits. This broad maximum might be attributable to the edge state. However, at a circular edge around the nanopit, both the zigzag and armchair edges inevitably coexist with com-

*Electronic address: hiroshi@phys.s.u-tokyo.ac.jp (a) zigzag edge

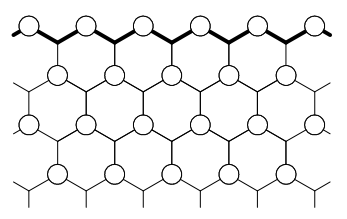

(b) armchair edge

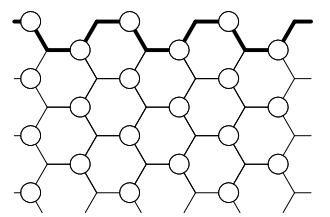

FIG. 1: Two graphite edges represented by the bold lines: zigzag edge (a) and armchair edge(b). The open circles are B-site carbon atoms.

parable probabilities, which makes it difficult to extract an electronic property for either of the edges. In another scanning tunneling microscopy (STM) measurement [7], a $(\sqrt{3} \times \sqrt{3}) R 30^{\circ}$ superstructure was observed near an armchair edge at a surface of highly oriented pyrolytic graphite (HOPG). So far, it is not clear whether similar superstructures appear near the zigzag edge or not [8].

In this report, we present results of STM and STS measurements for single step edges of both the zigzag and armchair types on a surface of exfoliated graphite, which contains much higher edge densities than non-exfoliated HOPG. With STS, we observed a peak in a tunnel spectrum at an energy just below $E_{F}$ on a terrace with the zigzag edge but not on that with the armchair one. This is the first clear spectroscopic observation of the theoretically predicted graphite edge state $[3,4]$. We also found two types of superstructures, i.e., the $(\sqrt{3} \times \sqrt{3}) R 30^{\circ}$ and honeycomb ones, coexisting on the terrace with both zigzag and armchair edges. Our calculations based on the density-functional derived non-orthogonal tight-binding model show that this coexistence is due to admixing of the two types of edges on the exfoliated graphite surface. 


\section{EXPERIMENTAL}

All the measurements shown here were made on ZYX exfoliated graphite (hereafter ZYX) 9]. ZYX was made from HOPG by graphite intercalation with $\mathrm{HNO}_{3}$ and by subsequent evacuation of the intercalant at high temperature. And then, it was evaculated at $1500{ }^{\circ} \mathrm{C}$ for $3 \mathrm{~h}$ to remove the remnant intercalants. The single crystallite size $(100-200 \mathrm{~nm}[10,11])$ is smaller than that in HOPG by an order of magnitude, which makes it much easier to find step edges on the surface with STM. All the graphite edges studied are monoatomic in height with an almost linear shape in the length scale of $100 \mathrm{~nm}$ or longer. We believe that active $\sigma$-orbital bonds at the edges are terminated by hydrogen or else since we did not try to remove them in ultra high vacuum (UHV) at elevated temperatures. Other details on the characterization of ZYX have been published elsewhere [9].

The STM images were obtained at room temperature in air with mechanically sharpened $\mathrm{Pt}_{0.8} \mathrm{Ir}_{0.2}$ wire tips. The data were acquired in the constant current mode with a tunnel current $(I)$ of $1.0 \mathrm{nA}$ and a bias voltage $(V)$ of $+0.1 \mathrm{~V}$ applied to the sample with respective to the tip. The STS data were obtained at $T=77 \mathrm{~K}$ in UHV of $P \leq 2 \times 10^{-7} \mathrm{~Pa}$ with our newly constructed STM [12]. A tunnel spectrum was obtained by averaging a set of $d I / d V$ vs. $V$ curves measured at 100 to 120 grid points over $5 \times 5$ to $10 \times 10 \mathrm{~nm}^{2}$ area by the lock-in technique $\left(f=71.73 \mathrm{~Hz}, V_{\bmod }=6 \mathrm{mV}\right)$.

\section{THEORETICAL MODELS}

In the calculations we assumed two layers of graphene for both types of edge structures. The bottom layer is composed of a graphene extended infinitely over the two dimensional plane, and is assumed to have no defects. The top layer with edge structures is prepared by removing a few tens to hundreds atoms from an infinite graphene. The lateral dimensions of the periodic supercells in the top layer are $12.8 \times 14.8 \mathrm{~nm}^{2}$ and $7.4 \times 25.6$ $\mathrm{nm}^{2}$ for the zigzag and armchair edges, respectively.

The electronic states of these graphite layers are calculated by the density-functional derived non-orthogonal tight-binding model [13]. We assumed that carbon atoms at the edges are completely hydrogen terminated, and took into account only the $\pi$-orbital at each carbon atomic site. These are relevant to the electronic states around $E_{F}$. LDOS at every atomic site is obtained by diagonalizing the Hamiltonian and overlap matrices generated from these $\pi$-orbitals at the $\Gamma$ point.

\section{RESULTS AND DISCUSSIONS}

An STM image taken near a zigzag step edge on the ZYX surface is shown in Fig. 2. Although an atomic resolution is not obtained right on the edge, we can identify it

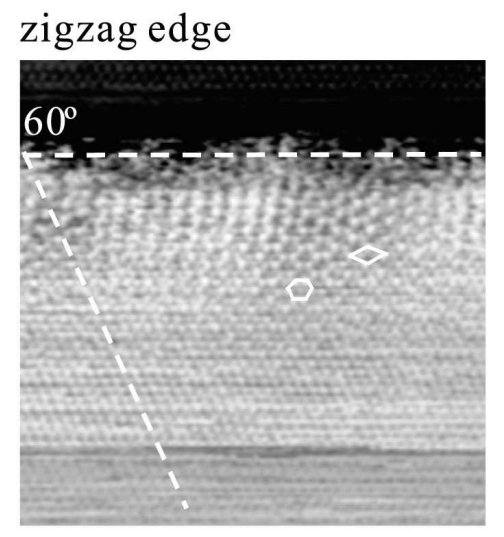

FIG. 2: An STM image near a zigzag step edge (the dashed line) on the surface of ZYX exfoliated graphite $(8 \mathrm{~nm} \times 8 \mathrm{~nm}$, $T=300 \mathrm{~K}$, in air). The long dashed line shows the atomic row of B-site atoms. The diamond and hexagon represent the $(\sqrt{3} \times \sqrt{3}) R 30^{\circ}$ superstructure and the honeycomb one, respectively.

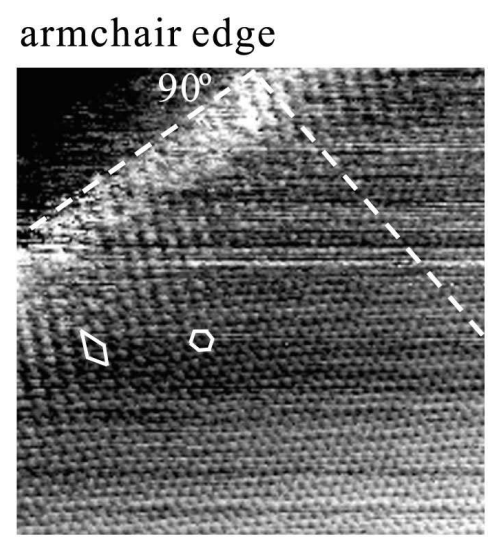

FIG. 3: An STM image near an armchair edge (the dashed line) on ZYX ( $8 \mathrm{~nm} \times 8 \mathrm{~nm}, T=300 \mathrm{~K}$, in air). The long dashed line shows the atomic row of B-site atoms. The diamond and hexagon represent the same meanings as in Fig. 2

as the zigzag type by noting that the atomic row of B-site carbon atoms (the long dashed line) on the upper terrace (the lower part of the image) is oriented at $60^{\circ}$ to the edge direction (the dashed line; see also Fig. 1(a)). The step height estimated from the line profile (not shown here) is $0.35 \mathrm{~nm}$, which is close to the graphite layer spacing $(=0.335 \mathrm{~nm})$. As seen in the figure, the edge is probably not a perfect straight line but has a slight irregularity. In other words, it might consist of dominant zigzag edges and a small fraction of armchair edges. Two types of superstructures are seen only on the upper terrace depending on the lateral position. One is the $(\sqrt{3} \times \sqrt{3}) R 30^{\circ}$ superstructure and the other is the honeycomb superstructure which consists of six B-site atoms. Typical spatial extensions of the superstructures are 3-4 $\mathrm{nm}$ from the edge. The superstructure pattern did not 
(a) zigzag edge

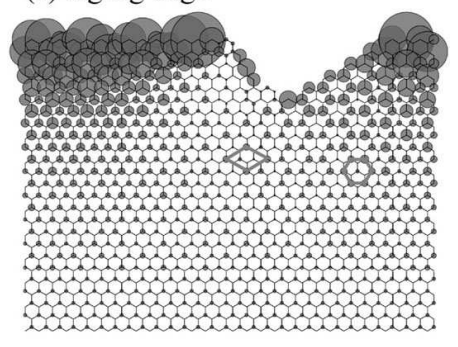

(b) armchair edge

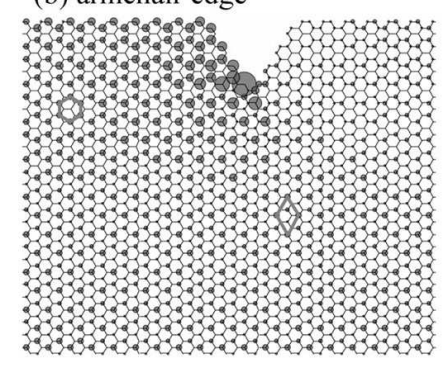

FIG. 4: Two examples of edge pattern used in the LDOS calculations: (a) a zigzag edge with a small amount of armchair edges and (b) an armchair edge with a small amount of zigzag edges. The radii of the circles plotted on the B-sites represent integrals of the calculated LDOS in an energy range between $E_{F}$ and $+100 \mathrm{meV}$. The diamond and hexagon represent the same meanings as in Fig. 2

depend on the bias voltage in a range between +0.05 and $+1.0 \mathrm{~V}$. This is probably the first STM image showing clearly the superstructures near the zigzag edge.

Figure B] is an STM image obtained near an armchair step edge. The edge type is identified from an angle $\left(=90^{\circ}\right)$ between the edge (the dashed line) and an atomic row of B-site atoms (the long dashed line). As was already reported by other workers [7], both the $(\sqrt{3} \times \sqrt{3}) R 30^{\circ}$ and the honeycomb superstructures are observed extending over $3-4 \mathrm{~nm}$ from the armchair edge. Therefore, it can be concluded that these superstructures appear both on the terraces with zigzag and armchair edges.

We now compare the experimental STM images with those simulated by calculations based on the densityfunctional derived non-orthogonal tight-binding model [13. In the case of the perfect zigzag edge, the calculations indicate that the electronic states are localized in the vicinity of the edge (decay length $\approx 0.5 \mathrm{~nm}$ ) and no superstructures appear anywhere. On the other hand, in the case of the perfect armchair edge, the calculations do not predict any localized states at the edge but instead a honeycomb superstructure persisting far beyond $5 \mathrm{~nm}$ from the edge. These calculated results for the perfect edges are inconsistent with our STM observations.

Hence, we have calculated the LDOS near the zigzag (armchair) edges which are mingled with small amounts of the armchair (zigzag) edges. Two examples of such

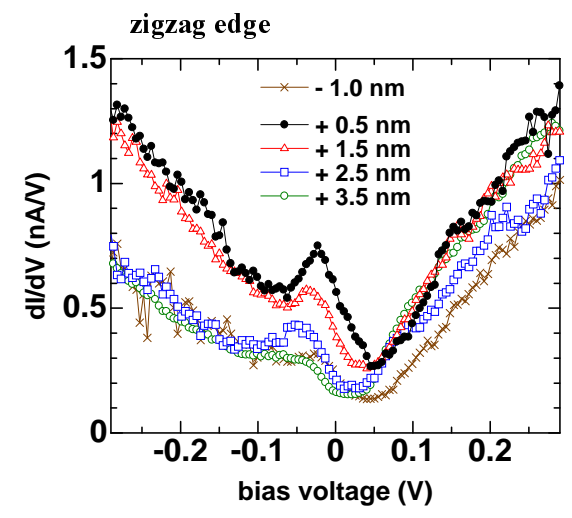

FIG. 5: $d I / d V$ vs. $V$ curves measured near a zigzag edge $(T=77 \mathrm{~K}$, in UHV). The numbers denoted are distances from the edge $(d=0)$.

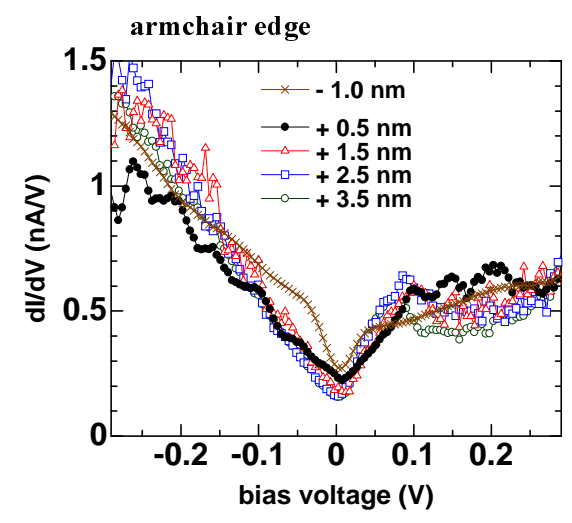

FIG. 6: $d I / d V$ vs. $V$ curves measured near an armchair edge $(T=77 \mathrm{~K}$, in UHV). The numbers denoted are distances from the edge $(d=0)$.

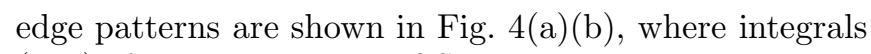
$\left(I_{\text {cal }}\right)$ of the calculated LDOS in an energy range between $E_{F}$ and $+100 \mathrm{meV}$ are represented by radii of the circles plotted on the B-sites. Note that $I_{c a l}$ should be proportional to the local tunnel currents at $V=+100 \mathrm{mV}$. As is seen in Fig. $4(\mathrm{a})$, both the $(\sqrt{3} \times \sqrt{3}) R 30^{\circ}$ and honeycomb superstructures appear on the terrace with the zigzag edges slightly mingled with the armchair edges. The superstructures have decay lengths of about $4-5 \mathrm{~nm}$ from the edge and rather complicated distributions in the parallel direction to the edge, which is consistent with the observation. Although the atomic arrangement of the zigzag edge in the experiment is not resolved clearly, the appearance of the two superstructures is strongly indicative that the edge in Fig. 2 is admixed with a small amount of the armchair edges.

The same is true for the other way around. Admixing of a small amount of the zigzag edges to an armchair edge can explain the experimental coexistence of the two superstructures. However, such admixing does not seem to explain the short decay lengths of the observed super- 
structures. The predicted long persistence of the honeycomb superstructure near the armchair edges is absent in Fig. 3. This is probably due to the three dimensional character of the experimental system, which is not fully taken into account in the present calculations. In any case, it is highly desirable to identify detailed edge shapes with atomic resolutions in the future.

Next, we show STS data taken near monoatomic step edges on ZYX obtained at $77 \mathrm{~K}$ in UHV. We chose the scan directions parallel to the edges, and $d I / d V$ curves taken at fixed distances $(d)$ from the edges were averaged to increase the signal to noise ratio. Observed corrugation amplitudes in the vicinity of the edges were rather position dependent both for the zigzag and armchair edges. This could be an additional indication of the mingling of the two types of edges. We took the spectroscopy data in Fig. 5] (6) at a position where the corrugation amplitude was the largest (smallest), based on the calculated results shown in Fig. 4 Fig. 5 shows tunnel spectra at different distances from a zigzag edge. A small but clear peak appears around $V=-20 \mathrm{mV}$ and grows as the tip approaches the edge on the terrace $(d>0)$. Note that the peak suddenly disappears when we move across the edge $(d=-1.0 \mathrm{~nm})$. A decay length of this structure, estimated by subtracting a smoothed base line from the spectra, is $1.6 \pm 0.4 \mathrm{~nm}$ which is in agreement with that $(1.2 \mathrm{~nm})$ for $I_{c a l}$ in Fig. 4(a). Since the tunnel current was unstable at $|d|<0.5 \mathrm{~nm}$ for some reason, we could not obtain reliable spectra right on the edge. It should also be noted that the definition of $d=0$ is somewhat arbitrary $( \pm 0.5 \mathrm{~nm})$ because of the absence of the atomic resolution in that region.

In contrast to the zigzag edge, we obtained qualitatively different spectra near an armchair edge as is shown in Fig. 6] where such a LDOS peak is not observed within the experimental scatters. Thus we conclude that the LDOS peak observed just below $E_{F}$ in Fig. [5]corresponds to the edge state that has been theoretically predicted to exist only for the zigzag edge [3, 4]. A recent firstprinciples calculation for a graphite ribbon with zigzag edges of $3 \mathrm{~nm}$ wide on an infinite graphene sheet [14] indicates that the LDOS peak due to the edge state is located below $E_{F}$ by a few tens $\mathrm{mV}$. This is in reasonable agreement with the peak in the tunnel spectra obtained in the present experiment.

\section{Acknowledgments}

One of us (H.F.) thanks the late Mitsutaka Fujita for stimulating his interest in the graphite edge state. The authors are grateful to $\mathrm{H}$. Akisato for useful comments on this manuscript. This work was financially supported by Grant-in-Aid for Scientific Research from from MEXT, Japan and ERATO Project of JST. One of us (T.M.) acknowledges JSPS Research Fellowship for Young Scientists.
[1] K.Tai and N. Shindo, Polym. Process. 35, 384 (1986).

[2] K. Kaneko, C. Ishii, M. Ruike, and H. Kuwabara, Carbon 30, 1075 (1991).

[3] M. Fujita, K. Wakabayashi, K. Nakada, and K. Kusakabe, J. Phys. Soc. Jpn. 65, 1920 (1996); K. Nakada, M. Fujita, G. Dresselhaus, and M. S. Dresselhaus, Phys. Rev. B 54, 17954 (1996); M. Fujita, M. Igami, and K. Nakada, J. Phys. Soc. Jpn. 66, 1864 (1997).

[4] Y. Miyamoto, K. Nakada, and M. Fujita, Phys. Rev. B 59, 9858 (1999).

[5] Z. Klusek, Z. Waqar, E. A. Denisov, T. N. Kompaniets, I. V. Makarenko, A. N. Titkov, and A. S. Bhatti, Appl. Surf. Sci. 161, 508 (2000).

[6] Z. Klusek, Vacuum 63, 139 (2001).

[7] P. L. Giunta and S. P. Kelty, J. Chem. Phys. 114, 1807 (2001).

[8] P. Esquinazi, A. Setzer, R. Höhne, C. Semmelhack, Y. Kopelevich, B. Kohlstrunk, and M. Lösche, Phys. Rev.
B 66, 024429 (2002).

[9] Y. Niimi, S. Murakawa, Y. Matsumoto, H. Kambara, and H. Fukuyama, Rev. Sci. Instrum. 74, 4448 (2003).

[10] R. J. Birgeneau, P. A. Heiney, and J. P. Pelz, Physica B 109\&110, 1785 (1982), and references therein.

[11] Y. Niimi, T. Matsui, H. Kambara, K. Tagami, M. Tsukada, and H. Fukuyama, to be published.

[12] T. Matsui, H. Kambara, and H. Fukuyama, J. Low Temp. Phys. 121, 803 (2000); T. Matsui, H. Kambara, I. Ueda, T. Shishido, Y. Miyatake, and H. Fukuyama, Physica B 329, 1653 (2003); H. Kambara, T. Matsui, and H. Fukuyama, to be published.

[13] M. Elstner, D. Porezag, G. Jungnickel, J. Elsner, M. Haugk, T. Frauenheim, S. Suhai, and G. Seifert, Phys. Rev. B 58, 7260 (1998).

[14] K. Tagami and M. Tsukada, to be published. 